\title{
Initial Clinical Experience of Pure Single-Incision Robotic Right Hemicolectomy with da Vinci SP Platform
}

\author{
Gyoung Tae Noh, M.D., Myunghyun Han, M.D., Soon Sup Chung, M.D., Ph.D., Ryung-Ah Lee, M.D., Ph.D., \\ Kwang Ho Kim, M.D., Ph.D. \\ Department of Surgery, Ewha Womans University College of Medicine, Seoul, Korea
}

The da Vinci SP Surgical System (dVSP; Intuitive Surgical, Sunnyvale, CA, USA) was introduced to perform pure single-incision surgery in 2018. This new surgical platform demonstrated favorable performance compared with the positive aspect of single-incision laparoscopic surgery and robot surgery. To date, its use has mainly been in urological and gynecological procedures. We report a case of successful robotic single-incision right hemicolectomy for cecal cancer with the dVSP.

Keywords: Robotic surgery, da Vinci SP, Single-incision, Right hemicolectomy

Supplementary video file: This article contains supplementary material (https://doi.org/ jmis.2019.22.4.181).
Received November 29, 2019

Revised December 8, 2019

Accepted December 9, 2019

Corresponding author

Kwang Ho Kim

Department of Surgery, Ewha

Womans University College of

Medicine, 260 Gonghang-daero,

Gangseo-gu, Seoul 07804, Korea

Tel: +82-2-6986-4689

Fax: +82-2-6986-4689

E-mail: eastgate@ewha.ac.kr

ORCID:

https://orcid.org/0000-0003-0928-6669

Video presentation at the 71th

Annular Congress of Korean

Surgical Society, Seoul, Korea, Oct.

31 to Nov. 2, 2019.

Copyright @ 2019 The Journal of Minimally Invasive Surgery. All rights reserved.

\section{INTRODUCTION}

Since Weber et al. ${ }^{1}$ reported the first procedure of robotic colon resection in 2002, robotic surgery had been verified as a safe and effective procedure for colorectal disease, with the advantage of stable and magnified three-dimensional view, increased degrees of freedom of devices, enhanced dexterity, superior ergonomic design, and a shorter learning curve. ${ }^{2-4}$ In 2018, Intuitive Surgical launched the da Vinci SP surgical system (dVSP). It was developed as a novel robotic platform to perform robotic single-incision surgery. It has double articulating joints in robotic instruments and articulating endoscope permitting flexible movement. All robotic instruments and endoscope are inserted through a cylindrical single port with a diameter of $2.5 \mathrm{~cm}$.

To date, its use has mainly been in urological and gynecological procedures. Herein, we report a case of cecal cancer, which was successfully operated with the dVSP.

\section{PROCEDURES}

\section{Patient}

A 65-year-old male diagnosed with cecal cancer on the 
screening colonoscopy, which showed a $2 \mathrm{~cm}$ sized fungating mass in cecum. Biopsy revealed it as a moderately differentiated adenocarcinoma. On the following computed tomography, no definite cecal wall thickening, metastatic lymph node or distant metastasis was identified, and a single-incision robotic right hemicolectomy with dVSP was planned. Due to the retrospective nature of this report and the use of an anonymized patient data, requirements for informed consent were waived by the IRB.

\section{Installation and docking}

The patient underwent standard bowel preparation the day before surgery and received prophylactic antibiotics before incision. A vertical midline incision, $35 \sim 40 \mathrm{~mm}$ in length, separating the umbilicus $10 \mathrm{~mm}$ away from the upper and lower margins of the umbilicus. An access port was made using a standard wound retractor of SurgiTractor ${ }^{\circledR}$ (SurgiCore, Gwangju, Korea) and size 6, non-latex, powder-free sterile glove. The surgical glove was fixed to the outer ring of the wound retractor. A $25 \mathrm{~mm}$ multichannel robotic trocar and two accessory assistant trocars $(12 \mathrm{~mm}$ and $5 \mathrm{~mm}$ ) were inserted through fingers of the surgical glove after cutting its fingertips. After achieving pneumoperitoneum with insufflations of carbon dioxide to $12 \mathrm{mmHg}$, the patient was placed in Trendelenberg position at $15^{\circ}$ and tilted left side down. After patients' positioning, laparoscopic exploration was undertaken using laparoscopic instruments through robotic and assistant ports. After completing laparoscopic exploration, the robot was docked with a $0^{\circ}$ flexible endoscope, monopolar curved scissors, bipolar grasper, and Cadiere forceps. All robotic endoscope and instruments were inserted through a $25 \mathrm{~mm}$ single robotic trocar.

\section{Surgical procedure}

Mobilization of the ascending colon and mesocolon was first performed via an inferior approach. The peritoneal reflection of the small bowel mesentery was incised. Dissection into the surgical plane above Gerota's fascia was performed to mobilize the entire ascending colon mesentery from the retroperitoneal structures. The duodenum and pancreatic head were exposed, with the hepato-duodenal ligament excised, and takedown of the hepatic flexure in a lateral to medial fashion. Lymphadenectomy was performed along the superior mesenteric vessels, with individual exposure and ligation of the ileocolic, right colic, and right branch of middle colic vessels at their origin from the superior mesenteric artery, vein, and middle colic trunk. The gastrocolic trunk was exposed and the colonic branch was ligated while preserving the pancreatic branches. Omentectomy was performed at the level inferior to the gastro-epiploic vessels and the transverse colon was mobilized from the omentum. Subsequently, the mesocolon of the transverse colon and the mesentery of the ileum were divided until the desired resection margins were achieved. Bowel resection and anastomosis were performed extracorporeally. After releasing the robot from docking and separating the glove from the wound retractor, mobilized bowel was exteriorized through an umbilical incision, and stapled ileocolic end-toside anastomosis was performed.

\section{Peri- and postoperative outcomes}

The procedure was successfully completed using a pure single-incision approach. Operative time, docking time, and console time were 355 minutes, 15 minutes, and 263 minutes, respectively. Estimated blood loss was $100.0 \mathrm{ml}$. Intraoperative complication or conversion to laparoscopic or open surgery was not occurred. On the pathologic report, $1.9 \mathrm{~cm}$ sized fungating mass infiltrating till proper muscle of cecum (pT2) was identified. Distance from tumor to the proximal and distal resection margins were $6.5 \mathrm{~cm}$ and $18 \mathrm{~cm}$, respectively. Twentythree lymph nodes were harvested and no metastatic lymph node was identified (pNO). The patient recovered without any complication and was discharged to home at postoperative day 6. Patient passed gas and resumed a soft diet on postoperative day 3.

\section{DISCUSSION}

The dVSP was designed for pure single-incision surgery with three double-jointed instruments and articulating endoscope inserted through one entry point. Double-jointed instruments enable the creation of adequate triangulation for surgical procedures. The single port enables $360^{\circ}$ anatomical access without port placement modification, thus overcoming the abdominal quadrant limitation of previous robotic platforms. To apply this new platform to colorectal surgery, we adopted a glove-port to secure a distance from the port to the surgical target. To achieve adequate triangulation with multijoint robotic instruments, the cannula tip should be $>12 \mathrm{~cm}$ from the surgical target to permit proximal joint passing the cannula tip and articulating. Using this glove-port and making the cannula tip to be outside of the abdominal cavity, we were able to secure the distance to the surgical target, especially for lymphadenectomy around the central vessels under trans-umbilical port placement, thus maximizing the cosmetic effect.

In the present report, the dVSP demonstrated favorable performance compared with positive aspect of single-incision 
laparoscopic surgery and robot surgery. There was minimal collision among instruments and endoscope compared to single-incision laparoscopic surgery while maintaining the advantages of robotic surgery such as better three-dimensional view, dexterity, and ergonomics. The peri- and postoperative results were also favorable. Considering its initial application for right hemicolectomy, the operation time was acceptable. Free location of robotic cart around operating table could be an advantage to shorten the docking time compared to previous robotic system models which required fixed location and angle to the operating table. Estimated blood loss during surgery and duration of postoperative hospital stay, time to first flatus and soft diet was comparable to previous reports of laparoscopic or robotic colorectal surgery. ${ }^{5-7}$ Regarding pathologic outcomes, the number of retrieved lymph nodes and status of resection margin were acceptable. The cosmetic result was satisfactory with a transumbilical incision, which was essential to extract the surgical specimen for colorectal surgery and visible scarring was minimal.

In summery, the dVSP is feasible and safe to perform single-incision robotic right hemicolectomy. It can maximize cosmetic effect while maintaining surgical principle of right hemicolectomy. Accumulation of data might confirm the validity of this surgical platform.

\section{ORCID}

Gyoung Tae Noh, https://orcid.org/0000-0001-9849-8211

Myunghyun Han, https://orcid.org/0000-0002-6541-8307

Soon Sup Chung, https://orcid.org/0000-0003-0921-8228

Ryung-Ah Lee, https://orcid.org/0000-0003-1146-3839

Kwang Ho Kim, https://orcid.org/0000-0003-0928-6669

\section{AUTHORS' CONTRIBUTIONS}

Conceptualization: GRN, GHK. Formal analysis: GTN, MHH, SSC, RAL, KHK. Methodology: GTN, MHH, SSC, RAL, KHK. Writing-original draft: GTN, KHK. Writing- review and editing: GTN, SSC, RAL, KHK.

\section{CONFLICT OF INTEREST}

None.

\section{FUNDING}

None.

\section{ACKNOWLEDGMENTS}

None.

\section{REFERENCES}

1) Weber PA, Merola S, Wasielewski A, Ballantyne GH. Teleroboticassisted laparoscopic right and sigmoid colectomies for benign disease. Dis Colon Rectum 2002;45:1689-1696.

2) Balch GC. Emerging role of laparoscopic and robotic surgery for rectal cancers. Ann Surg Oncol 2009;16:1451-1453.

3) Pigazzi A, Garcia-Aguilar J. Robotic colorectal surgery: for whom and for what? Dis Colon Rectum 2010;53:969-970.

4) Rashid TG, Kini M, Ind TEJ. Comparing the learning curve for robotically assisted and straight stick laparoscopic procedures in surgical novices. Int J Med Robot 2010;6:306-310.

5) Rondelli F, Balzarotti R, Villa F, et al. Is robot-assisted laparoscopic right colectomy more effective than the conventional laparoscopic procedure? A meta-analysis of short-term outcomes. Int J Surg 2015;18:75-82.

6) Kim MJ, Park SC, Park JW, et al. Robot-assisted Versus Laparoscopic Surgery for Rectal Cancer: A Phase II Open Label Prospective Randomized Controlled Trial. Ann Surg 2018;267:243251.

7) Park EJ, Cho MS, Baek SJ, et al. Long-term oncologic outcomes of robotic low anterior resection for rectal cancer: a comparative study with laparoscopic surgery. Ann Surg 2015;261:129-137. 